\title{
KONZEPT GENDER THESAURUS. ZUR BEDEUTUNG EINER GE- MEINSAMEN DOKUMENTATIONSSPRACHE FÜR FORSCHUNG UND INFORMATIONSEINRICHTUNGEN
}

\author{
von Jasmin Schenk
}

Zusammenfassung: Angesichts aktueller und voraussichtlich in naher Zukunft startender Infrastrukturprojekte der Frauen/Gender-Informationseinrichtungen einerseits und der Gender Studies andererseits, soll im Folgenden auf den Sinn und die Notwendigkeit einer gemeinsamen gendersensiblen Dokumentationssprache hingewiesen werden. Dieser Beitrag entstand im Rahmen des Masterstudiums der Bibliotheks- und Informationswissenschaften (MALIS) an der Technischen Hochschule Köln und verfolgt das Ziel, Vorschläge für die Erarbeitung eines Gender Thesaurus zu entwickeln.

Schlüsselwörter: Gender Thesaurus; gendergerechte Sacherschließung; feministische Sprachkritik; i.d.a.-Dachverband; META-Katalog; Repositorium Gender Studies; Digitales Frauenarchiv; Thesaurusmanagement

\section{GENDER THESAURUS CONCEPT : ON THE SIGNIFICANCE OF SHARING A SPECIFIC GENDER-SENSITIVE LANGUAGE OF DO- CUMENTARY}

Abstract: In the light of current and prospective projects of women's /gender information centres on the one hand and gender studies institutions on the other, the article focuses on the significance and the necessity of sharing a specific gender-sensitive language of documentary. Written in the process of the Master's program Library and Information Science (MALIS) at the TH Köln, the intention of this paper is to formulate suggestions as to how a gender thesaurus could be modelled.

Keywords: gender thesaurus; gender-sensitive indexing; feminist language criticism; i.d.a.-umbrella organization of German-language archives and libraries of the Women's and Lesbian Movements; META-catalog; gender studies repository; digital women's archive; thesaurus management

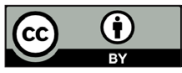




\section{Frauen-/gendersensible Sacherschließung}

In den 1990er Jahren machte Dagmar Jank frauengerechte Sacherschließung ${ }^{1}$ und „Frauenthesauri im Informations- und Dokumentationsbereich “2 zum Thema der fachlichen Diskussion, musste aber schnell feststellen, dass „der Versuch, die frauengerechte Sacherschließung in bundesdeutschen Bibliotheken voranzutreiben", gescheitert war. ${ }^{3}$ In diesen Zeitraum fallen auch die Publikationen erster deutschsprachiger feministischer Thesauri.

Die Pflege von eigenen Normdaten seitens der Frauen-/Genderinformationseinrichtungen ${ }^{4}$ ist erforderlich, weil die traditionellen bibliothekarischen Erschließungssysteme (insbesondere die GND) in Bezug auf gendergerechte Sacherschließung bis heute eklatante Lücken und Asymmetrien aufweisen, die auf dichotomen Geschlechterstereotypen und dem Prinzip des generischen Maskulinums beruhen. ${ }^{5}$ Karin Aleksander greift in einem aktuellen Artikel mit dem Titel „Die Frau im Bibliothekskatalog“ einige Beispiele von Jank wieder auf und belegt, dass auch nach über zwanzig Jahren noch Handlungsbedarf besteht: „Wenn über die Qualität von Katalogen diskutiert wird, sollte das Potential, das eine gendersensible Verschlagwortung bietet, genutzt werden. Dazu brauchen wir mehr Analysen, die Lücken, Fallen und Fehler in den bisherigen Systematiken und Klassifikationen auf der Grundlage der Ergebnisse der Geschlechterforschung aufzeigen. “6

Ein eigener Gender Thesaurus ist also nicht nur Zugangsvokabular für die Recherche in den thematisch spezifischen, interdisziplinären Beständen der Frauen-/Genderinformationseinrichtungen, sondern auch ein politisches Instrument feministischer/genderbewusster Sprachkritik im Allgemeinen und an androzentristisch geprägten bibliothekarischen Dokumentationssprachen im Besonderen.

\section{Relevante Projekte und Einrichtungen}

Kernziel dieses Beitrags ist es, eine theoretische Grundlage für die mögliche Kooperation zweier Projekte zu entwickeln: 1) META-Katalog des i.d.a.-Dachverbands ${ }^{7}$ und 2) Einrichtung eines Fachrepositoriums für die Geschlechterforschung.

Seit Oktober 2012 arbeiten die Einrichtungen des internationalen Dachverbandes der deutschsprachigen FrauenLesbenarchive, -bibliotheken und -dokumentationsstellen i.d.a. (informieren, dokumentieren, archivieren) am gemeinsamen META-Katalog, der vom deutschen Bundesministerium für Familie, Senioren, Frauen und Jugend (BMFSFJ) gefördert wird und im 
vergangenen Herbst online gestellt wurde (siehe meta-katalog.eu). Ziel ist der Aufbau einer zentralen Nachweisdatenbank zur Bündelung von genderspezifischem Wissen und zur Sichtbarmachung jahrzehntelang gesammelter Bestände von 36 deutschsprachigen Frauen- und Genderbibliotheken und -archiven. ${ }^{8}$ Der i.d.a.-Dachverband begegnet damit den Nutzungserwartungen, indem die heterogenen Bestandsdaten aus unterschiedlichen Erschließungssystemen auf eine möglichst homogene Art und Weise in einem modernen Discovery System (VuFind) zugänglich gemacht werden.

Im Mai 2015 haben drei kooperierende Institutionen (Freie Universität Berlin, Humboldt-Universität zu Berlin und Technische Universität Berlin) einen DFG-Antrag zur Einrichtung eines Open-Access-Repositoriums für Gender Studies eingereicht. Es handelt sich dabei um eine Empfehlung der DFG nach Abschluss des FU-Projektes „Geschlechterforschung und Open Access“ aufgrund des „nach wie vor bestehenden Entwicklungsrückstands der Geschlechterforschung im Umgang mit neuen Publikationsmöglichkeiten" .

Eine weitere Perspektive, die an dieser Stelle berücksichtigt und mitgedacht werden soll, stellt das 2013 im Koalitionsvertrag der Bundesregierung formulierte Vorhaben zur Einrichtung eines digitalen Frauenarchivs dar: „Wir wollen die wissenschaftliche Aufarbeitung der Deutschen Frauenbewegung, unter besonderer Beachtung der Frauenbewegung in der DDR und der Umbruchzeit 1989/90 vorantreiben, indem wir die existierenden Materialien unter Einbeziehung der Frauenarchive in einem 'Digitalen Deutschen Frauenarchiv' sichern und der Öffentlichkeit zugänglich machen."10

Der i.d.a.-Dachverband sieht sich in der Verantwortung, aufbauend auf dem META-Projekt, die umfassende Fachkompetenz der Mitgliedseinrichtungen in die Ausgestaltung eines solchen digitalen Frauenarchivs einzubringen. Mit dem META-Katalog existiert bereits ein Nachweissystem für die relevanten, vielseitigen Bestände zur Frauenbewegung und Geschlechtergeschichte, das in einem ersten Schritt durch Digitalisate angereichert werden kann. Perspektivisch gesehen bietet sich damit die einzigartige Möglichkeit, ein Fachportal aufzubauen, das nicht nur wertvolles Forschungsmaterial anschaulich verfügbar machen, sondern als zentraler Aggregator auch Zulieferer für die Deutsche Digitale Bibliothek und Europeana werden kann.

Die genannten Projektvorhaben stellen, nicht zuletzt durch bestehende Kontakte und personelle Überschneidungen, im Falle ihrer Realisierung und Verstetigung die Grundlage für eine zukunftsorientierte Informationsinfrastruktur für die interdisziplinäre Genderforschung dar. Zudem bestehen bereits internationale Netzwerke, durch die in der Vergangenheit er- 
folgreich Kooperationsprojekte ${ }^{11}$ realisiert wurden und welche die Umsetzung weiterer Folgeprojekte auf europäischer Ebene nahelegen:

- Der deutschsprachige i.d.a-Dachverband, zu dem Einrichtungen aus Deutschland, Österreich, Luxemburg, Italien und der Schweiz gehören, ist Mitglied im Women's Information Network of Europe $(\text { WINE })^{12}$

- ATGENDER: The European Association for Gender Research, Education and Documentation ist die internationale Fachgesellschaft von Organisationen der Frauen- und Genderforschung sowie der Informationseinrichtungen von WINE ${ }^{13}$

- Das European Institute for Gender Equality (EIGE) ist eine EU-Einrichtung mit Sitz in Vilnius, Litauen, und verfügt über ein eigenes Resource \& Documentation Centre (RDC), für dessen Datenbank i.d.a.- und WINE-Einrichtungen themenspezifisch (z.B. zu Gleichstellungsfragen, geschlechterbasierter Gewalt, weiblicher Genitalverstümmelung etc.) relevante Bestandsdaten geliefert haben (Genderbibliothek des ZtG der Humboldt-Universität zu Berlin für Deutschland, Cid-femmes et genre für Luxemburg). ${ }^{14}$ EIGE plant gegenwärtig ebenfalls zur Vereinheitlichung der Terminologie mit anderen EU-Institutionen einen Thesaurus für europäische Gleichstellungspolitik aufzubauen ${ }^{15}$.

Es empfiehlt sich daher in engem Erfahrungsaustausch die Thesauri so zu modellieren, dass zukünftig eine Verknüpfung realisiert werden kann.

\section{Konzeptentwickung}

Die Einrichtungen des i.d.a.-Dachverbands treffen sich jährlich zu einer mehrtägigen Arbeitstagung, deren fester Bestandteil eine Arbeitsgruppe "Systematik“ ist. Zweck dieser AG ist es, gemeinsam Fragen der Sacherschließung zu diskutieren. Beim Treffen 2013 in Leipzig berichtete Karin Aleksander (Leiterin der Genderbibliothek des Zentrums für transdisziplinäre Genderstudien der Humboldt-Universität zu Berlin) von den Plänen zur Einrichtung eines Fachrepositoriums Gender Studies. Für die systematische Erschließung von Open Access-Publikationen ist ebenso wie für die Recherche in der META-Nachweisdatenbank eine gendersensible Dokumentationssprache erforderlich.

Bei der 12. Arbeitstagung der „Konferenz der Einrichtungen für Frauenund Geschlechterstudien im deutschsprachigen Raum (KEG)“ in Pader- 
born im Februar 2014 wurden beide Projekte in einer AG mit dem Titel "Gender goes online? Gender Wissen im Netz" den Einrichtungen der Gender Studies bekannt gemacht. Als Ergebnis wurde festgehalten, dass es für die Sicherstellung der Literaturversorgung der inter- und transdisziplinären Gender Studies einer kontinuierlichen Zusammenarbeit zwischen Frauen-/Genderinformationseinrichtungen und den Lehrenden des Faches bedarf, da sich beide Seiten mit gleichen Herausforderungen konfrontiert sehen (z.B. dem Entgegenwirken der Wissenskanonisierung in allgemeinen Bibliotheken und deren Erschließungssystemen). ${ }^{16}$

Daher erschien es naheliegend, über eine gemeinsame Systematik bzw. einen Thesaurus nachzudenken, der im Hinblick auf zukünftige Anwendungsszenarien idealerweise den Anforderungen für die Interoperabilität von Metadaten entspricht. So entstand die Idee, basierend auf der Analyse vorhandener Thesauri und Schlagwortkataloge der i.d.a.-Einrichtungen einen interdisziplinären Gender Thesaurus zu entwickeln, dessen Verbreitung in Informationssystemen der Frauen-/Genderinformationseinrichtungen und der Gender Studies gleich mehrere innovative Chancen mit sich bringt:

- Es wird eine Brücke geschlagen zwischen den Informationseinrichtungen und den Forschungseinrichtungen der Gender Studies bei gleichzeitiger Verwendung des Gender Thesaurus im geplanten Gender Repositorium.

- Die Vereinheitlichung der bisher heterogenen Schlagwortlisten und Thesauri, die in den Frauen/Genderinformationseinrichtungen zur Sacherschließung zum Einsatz kommen, wird vorangetrieben.

- Einrichtungen, die bisher nur unzureichend oder gar nicht inhaltlich erschließen (können), bekommen ein wirksames Werkzeug zur Hand.

- Das Information Retrieval im META-Nachweiskatalog wird optimiert.

- Ein Gender Thesaurus bietet Chancen, über Debatten in Fachpublikationen hinaus, Referenzsystem für allgemeine Klassifikationen, Schlagwortnormdateien und Thesauri zu werden, um damit auch in sonstigen Bibliotheken und Archiven genderrelevante Bestände besser sichtbar und auffındbar machen zu können.

\section{Methodische Umsetzung}

Neben dem fachlichen Austausch bei Tagungen und Arbeitstreffen des META-Fachbeirats sowie einer ausführlichen Literaturauswertung sollte der 
Vergleich von relevanten Thesauri und Schlagwortkatalogen die Grundlage für die Erarbeitung eines Konzeptes bilden, welches dem META-Projekt zur Verfügung gestellt werden soll. Im Verlauf der Analyse stellte sich heraus, dass die bestandsspezifischen Vokabulare, die daran ersichtlichen unterschiedlichen feministischen Positionen sowie die variierenden fachlichen Kapazitäten der i.d.a.-Einrichtungen das Vorhaben der Vereinheitlichung erschweren. Einen vorhandenen feministischen Thesaurus als Grundlage zu nehmen, erwies sich daher als genauso wenig zielführend, wie die zeitintensive gemeinsame Erarbeitung einer grundlegenden Neufassung. Es galt daher alternative Lösungsansätze zu entwickeln, wie entweder ein vereinfachter Basis-Thesaurus basierend auf den Gemeinsamkeiten der vorhandenen Vokabulare generiert oder alternativ eine deutsche Fassung eines etablierten internationalen Frauenthesaurus erstellt werden kann.

\section{Themenüberblick}

Abtreibung Afrika Arbeit Armut BRD Berlin Beruf Bildung Biographie Bundesrepublik Deutschland Christentum DDR DeutsChland Diskriminierung Ehe Emanzipation Erwerbsarbeit Erwerbstätigkeit Erziehung Europa Familie FeminismUS Feministische Theorie Film

Frankreich Frauenarbeit Frauenbewegung Frauenbewegung und -projekte Frauenbild Frauenforschung Frauentorderung Frauenorganisation Frauenpolitik Fravenrechte Gender GeSChichte

Geschlechterdifferenz Geschlechterforschung Geschlechterrolle

Geschlechterverhältnis Gesundheit Gewalt Gewalt gegen Frauen Gleichberechtigung Gleichstellung Großbritannien Hausarbeit Hochschule Homosexualităt Identităt Indien Interview Islam Italien Karriere Krieg Krieg Militarismus Kultur Kunst Körper Künstlerin Lebenslauf Lesbe Lesben Liebe Literatur Macht

Medien Medizin Menschenrechte.Mexiko Migrantin Migration Musik Mutterschaft

\section{Mädchen Nationalsozialismus} Neue Frauenbewegung Philosophie $\mathrm{PO}$ it ik Politikerin Prostitution Psychoanalyse Psychologie Rassismus Recht Religion Schriftstellerin Schule schweiz sexismus SexUalität sexuelle Gewalt sport USA Vereinigte Staaten von Amerika Vergewaltigung Weiblichkeitsbild widerstand

\section{Österreich}

Abb. 1: Schlagwortwolke im META-Katalog 


\section{Thesauruserstellung}

Die Einspielung der Bestandsdaten von Mitgliedseinrichtungen des i.d.a.Dachverbands in ein gemeinsames System bietet die einmalige Chance, vorhandene Schlagworte miteinander zu vergleichen und das Vorgehen zu automatisieren. Da die Erschließungsmethoden und die Datenqualität sehr heterogen sind, werden vorab die individuellen Erfassungsmasken vom Projektteam analysiert und ohne standardisierte bibliothekarische Austauschformate (MARC21) nur in der Auszeichnungssprache XML über eine Datenumwandlung mittels XSLT in VuFind ${ }^{17}$ importiert. Die Datenmodellierung von XML-Daten erfolgt nach einem eigens entwickelten METAArbeitsformat, welches die spätere Ausgabe in standardisierten Austauschformaten möglich machen soll (MARC21, EAD, DublinCore etc.).

Für die Erarbeitung eines Thesaurus relevant sind die folgenden Felder des META-Arbeitsformats: subjectTopic/ subjectPerson / subjectGeographic. Es können alphabetische Listen dieser Felder für jede beteiligte Einrichtung ausgegeben werden, die als Grundlage für den automatischen Datenabgleich und zur Modellierung des gemeinsamen Thesaurus dienen. Bei diesem Verfahren handelt es sich nicht um ein Mapping von kontrollierten Vokabularen aufeinander, sondern lediglich um den Abgleich von Schlagwortlisten, die gewissermaßen als „Wortgutsammlung“ ${ }^{8}$ für die Thesauruserstellung dienen. Je höher die Übereinstimmungen beim Abgleich der Wortlisten sind, desto geeigneter ist ein Begriff dann als Deskriptor für den Gender-Thesaurus. Diese können dann mit einer geeigneten Thesaurus-Management-Software zu einem Gender Thesaurus modelliert werden, der aufgrund der Interdisziplinarität der Gender Studies zwangsläufig ein hohes Maß an polyhierarchischen Relationen aufweisen wird. Ein solcher intellektueller Vorgang setzt eine Institutionalisierung der Thesaurusverwaltung für META (und Kooperationspartnerlnnen) voraus, die nur möglich ist, wenn das Projekt verstetigt wird.

Alternativ könnte auch der Versuch unternommen werden, vorhandene feministische Thesauri zu vereinen bzw. zu verknüpfen. Vorhandene Thesauri können aus dem verbreiteten System FAUST auch im XML-Format wahlweise alphabetisch oder systematisch ausgegeben werden (Abb. 2).

Es wäre interessant, das Maß an Übereinstimmungen zwischen den vorhandenen Thesauri der i.d.a.-Einrichtungen, die mit FAUST arbeiten, über ein automatisiertes Mapping zu überprüfen und die Ergebnisse mit dem oben geschilderten Verfahren zu vergleichen. Bei dem beschriebenen Mapping entstünde eine Crosskonkordanz zwischen den jeweiligen Thesauri. „Crosskonkordanzen sind gerichtete relevanzbewertende Relationen 
zwischen Termen zweier Thesauri oder Klassifikationen. Sie ermöglichen die Übersetzung von Anfragetermen deines Erschließungssystems in ein anderes." 19

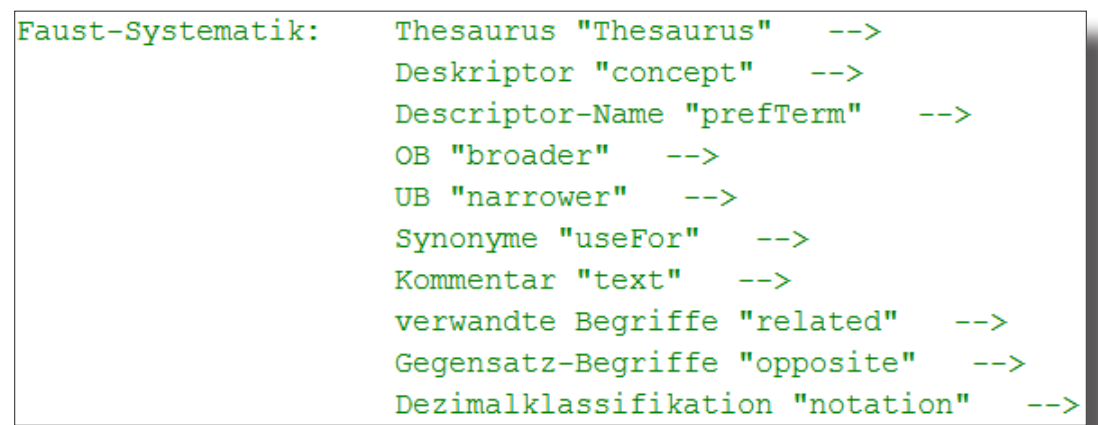

Abb. 2: XML-Schema bei Thesaurus-Export aus FAUST

Als problematisch könnten sich dabei konzeptuelle Unterschiede erweisen, da beispielsweise die österreichische ThesaurA erwartungsgemäß ein höheres Maß an präkoordinierten Deskriptoren aufweist ${ }^{20}$ als deren Vorgänger, der Feministische Thesaurus des FrauenMediaTurm ${ }^{21}$. Außerdem sind in einigen Themenfeldern politische Diskussionen über Vorzugsbenennungen zu erwarten (Bsp. Prostitution vs. Sexarbeit).

Die Erstellung einer Konkordanz könnte auch einen mehrsprachigen Thesaurus als Ergebnis haben. 1992 brachte das damalige Informatiecentrum en Archief voor de Vrouwenbeweging (IIAV) in Amsterdam, heute ATRIA - Institute of Gender Equality and Women's History, den niederländischen Vrouwenthesaurus heraus. ${ }^{22}$ Auf dessen Basis wurde 1998 der European Women's Thesaurus (EWT) in Zusammenarbeit mit vier weiteren Institutionen entwickelt: KVINFO Centre for Information on Women and Gender in Kopenhagen, RoSa Documentation Centre in Brüssel, Bibliotheca/Centro di Documentazione delle Donne in Bologna und dem Nordic Institute for Women's Studies and Gender Research (NIKK) in Oslo. ${ }^{23}$ Beide Thesauri werden bis heute von Atria gepflegt und stehen online zur Recherche zur Verfügung.

Obwohl der Eindruck entstehen könnte, als seien es zwei unterschiedliche Thesauri, handelt es sich beim EWT tatsächlich um eine Übersetzung des Vrouwenthesaurus, der in einem separaten System gepflegt und in den jeweiligen Kooperationseinrichtungen an deren individuelle Anforderungen angepasst wird. Es gibt einen gemeinsamen, XML-basierten „Basis-Thesaurus" bei Atria, der aber nicht zentral für alle Einrichtungen gleichermaßen 
administriert wird, sondern national beliebig erweitert werden kann. Die Verknüpfung eines deutschsprachigen Thesaurus mit dem englischsprachigen EWT würde sich für Kooperationen mit Atria bzw. WINE als äußerst nützlich erweisen und würde aufgrund der Verbreitung mehr fachliche Beachtung erlangen. Voraussetzung für so ein ambitioniertes Projekt wäre aber die zentrale Administration und die Bereitstellung in einem interoperablen Metadatenschema, damit nicht mehrere unterschiedliche Varianten verwendet werden und die Verknüpfung mit allgemeinen Vokabularen angestrebt werden kann.

\section{Fazit und Ausblick}

Um das Information Retrieval im META-Katalog zu optimieren, wäre die Erstellung eines Gender Thesaurus wünschenswert, der sich aber nur bei einer Fortsetzung und Verstetigung des Projekts realisieren lässt. In einem ersten Schritt könnte dann, basierend auf den in META zusammengeführten Schlagwortlisten der einzelnen i.d.a.-Einrichtungen, der Grundstock eines gemeinsamen Thesaurus gebildet werden. Dieser Basis-Thesaurus kann dann in META zusätzlich zu den verbleibenden (vom Thesaurus nicht berücksichtigten) freien Schlagworten zur Recherche angeboten werden. So wird die Vereinheitlichung gefördert und Nutzenden ein kontrolliertes Vokabular angeboten, ohne den Verlust von Informationen hinnehmen zu müssen, da die spezifischen Besonderheiten der Sacherschließung zusätzlich erhalten blieben. Je nach Kapazität wäre es wünschenswert, wenn die Vereinheitlichung der Schlagworte nicht nur in META passieren, sondern auch zurückgeführt werden und schrittweise die unterschiedlichen Vokabulare der Lokalsysteme von der Standardisierung profitieren würden ${ }^{24}$. Parallel dazu kann, der Empfehlung von Marianne Boere (Atria, Amsterdam) folgend ${ }^{25}$, die bereits existierende i.d.a.-Kommunikationsplattform von der Arbeitsgruppe Systematik zur Diskussion terminologischer Probleme genutzt werden.

Technisch gesehen kann das META-Team auf umfangreiche Forschungsergebnisse zur Interoperabilität von Metadaten, zur Heterogenitätsbehandlung in der Sacherschließung durch Einsatz von Crosskonkordanzen ${ }^{26}$ und auf die Entwicklung von Datenmodellen für das Semantic Web zurückgreifen. Die aktuelle fachliche Diskussion findet auf fortgeschrittener Ebene statt. Grundlage sind überwiegend bereits etablierte Thesauri, die zur Optimierung des Information Retrieval bei paralleler Recherche in heterogenen Datenbeständen von Fachportalen miteinander verknüpft wer- 
den. Vorteil dieser Forschungslage ist, dass bei Detailfragen die entsprechende Expertise eingeholt werden kann und dass Open-Source Tools für das Thesaurusmanagement ${ }^{27}$ zur Verfügung stehen.

Obwohl es an dieser Stelle vornehmlich um die Entwicklung eines deutschsprachigen Zugangsvokabulars geht, bietet sich perspektivisch eine Zusammenführung auf europäischer Ebene an, die durch eine Konkordanz zum EWT realisiert werden kann.

Obwohl das Modellprojekt inzwischen eingestellt wurde, wäre es sicher interessant, die Verantwortlichen des Theologischen Schlagwortkatalogs für Gender Forschung (TSG) ${ }^{28}$ an der Ruhr-Universität Bochum zu befragen, ob das Projekt zur Aufbereitung des Schlagwortkatalogs für das Social Web nach Möglichkeit weiterverfolgt werden soll, welche Erfahrungen sich daraus ziehen lassen und was ggf. für META nachgenutzt werden könnte. Die Bereitstellung des Schlagwortkatalogs als Linked Open Data basierend auf dem SKOS-Datenmodell ${ }^{29}$ sollte Grundlage für die Verknüpfung mit weiteren Projekten sein. So hatte die Verfasserin im Frühjahr 2013 Kontakt zu der TSG-Projektmitarbeiterin Miriam Heise (RUB Bochum), die eine Verknüpfung zwischen dem TSG und dem Feministischen Thesaurus des FrauenMediaTurm anregen wollte. Der FMT-Sachthesaurus wurde dem Projekt in einer XML-Datei zur Verfügung gestellt, aber eine Verknüpfung konnte mangels Kapazität nicht realisiert werden.

Um von den Detailfragen der Thesaurusentwicklung zurück zum strategischen Ziel zu kommen, nämlich der Optimierung der Literaturversorgung für die Gender Studies durch die Vernetzung von Infrastrukturprojekten auf Basis eines gemeinsamen Vokabulars, soll zum Abschluss auf einen Beitrag im Themenheft „Digitalisierung zwischen Utopie und Kontrolle“ der Zeitschrift Femina Politica hingewiesen werden. Darin beschreiben Ulrike Koch und Anna Zschokke ihre Utopie eines queer-feministischen Metaarchivs im Internet, welches sowohl die relevante digitale Wissensproduktion (Blogs, Tweets, Fotos etc.) als auch die „Bewegungsmaterialien“ der bestehenden feministischen Archive und Bibliotheken umfassend sichern soll. ${ }^{30}$ Dabei erwähnen sie durchaus den i.d.a.-Dachverband, scheinen aber zur der Zeit das META-Projekt noch nicht zu kennen. Sollten die anderen, oben beschriebenen Projekte im Sinne des Dachverbandes umgesetzt werden, gäbe es ein Digitales Frauenarchiv auf Basis des META-Katalogs, das idealerweise auch durch eine Kooperation mit dem Repositorium der Gender Studies verknüpft würde. Damit wäre eine Infrastruktur geschaffen, die bereits einen Großteil der o.g. Forderungen erfüllen und auf der durch weitere Kooperationen aufgebaut werden kann. Entscheidende Voraussetzung dafür ist, dass im Konzept eines Digitalen Frauenarchivs die sogenannten 
„digital born documents" von Beginn an mitgedacht und auch internationale Einrichtungen (insbesondere Mitglieder des i.d.a-Dachverbands) eingebunden werden.

Jasmin Schenk M.A. FrauenMediaTurm - Das Archiv und Dokumentationszentrum Bayenturm, D-50678 Köln E-Mail: schenk@frauenmediaturm.de

Jasmin Schenk hat Kunstgeschichte und Anglistik mit interdisziplinärem Studienschwerpunkt der Frauen- und Geschlechterforschung (Universität Trier, Abschluss Magistra 2004) studiert und ist wissenschaftliche Leiterin des FrauenMediaTurm (FMT). Seit 2006 vertritt sie den FMT im i.d.a.-Dachverband, wurde 2012 in den Fachbeirat von dessen META-Projekt berufen und zählt derzeit zum Fachbeirat des Deutschen Digitalen Frauenarchivs. Aktuell bereitet sie sich außerdem auf ihre Masterarbeit im Studiengangs Bibliotheks- und Informationswissenschaft (MALIS) an der TH Köln vor.

\section{Literatur und Quellen}

Letztes Abrufdatum der folgenden Internet-Dokumente ist der 30.01.2016.

Aleksander, Karin 2014: Die Frau im Bibliothekskatalog. In: LIBREAS. Library Ideas, 25. http://libreas.eu/ausgabe25/02alexander/.

ATGENDER: The European Association for Gender Research, Education and Documentation: http://www.atgender.eu/.

Bertram, Jutta 2005: Einführung in die inhaltliche Erschließung. Grundlagen - Methoden - Instrumente. Würzburg: Ergon, 2005.

Boere, Marianne; Drenthe, Gusta 1998: European women's thesaurus. A structured list of descriptors for indexing and retrieving information in the field of the position of women and women's studies. Amsterdam: International Information Centre and Archives for the Women's Movement (IIAV).

Deutschlands Zukunft gestalten. Koalitionsvertrag zwischen CDU, CSU und SPD, 2013, S. 103, https://www.bundesregierung.de/Content/ DE/StatischeSeiten/Breg/koalitionsvertrag-inhaltsverzeichnis.html.

Drenthe, Gusta; Sommen, Maria van der; Boere Marianne 1992: Vrouwenthesaurus. Lijst van gecontroleerde termen voor het ontsluiten van informatie over de positie van vrouwen en vrouwenstudies. Amsterdam: 
Internationaal Informatiecentrum en Archief voor de Vrouwenbeweging (IIAV); Anna Maria van Schuurman Centrum (AMSC).

EIGE, Resource \& Documentation Centre (RDC): http://eige.europa.eu/ rdc.

EIGE, Agenda des Meetings vom 25.-26. Februar 2015 in Brüssel. http://eige.europa.eu/sites/default/files/Draft-Agenda-EIGERDCmeeting\%20on\%20glossary\%20and\%20thesaurus-16Feb2015.pdf.

Gesis-Forschung: http://www.gesis.org/forschung/drittmittelprojekte/archiv/komohe/.

Jank, Dagmar 1991: Die Nicht-Gleichbehandlung von Frauen und Männern in der Schlagwortnormdatei. Ein offener Brief. In: Bibliotheksdienst 25, 1991, H 9, S. 1418-1421.

Jank, Dagmar 1992: Die Frauenfrage und die Sacherschließung. In: Laurentius 9, 1992, H 1, S. 56-59.

Jank, Dagmar 1996: Frauenthesauri im Informations- und Dokumentationsbereich. In: Bibliotheksdienst 30, 1996, H. 11, S. 1913-1920.

Jong, Sarah de; Meulmeester, Gé; Vriend, Tilly 2013: Core feminist texts in Europe online: teaching with the FRAGEN database. In: Teaching gender with libraries and archives. Jong, Sarah de (Hrsg.). Budapest, New York: Central European University Press. S. 76-98.

Konferenz der Einrichtungen für Frauen- und Geschlechterstudien im deutschsprachigen Raum (KEG): Protokolle aus den Arbeitsgruppen der KEG 2014: http://www.genderkonferenz.eu/downloads/2014/ AG_Protokolle KEG 2014_30_4_14.pdf.

Klösch-Melliwa, Helga; Zach, Angelika 1996: ThesaurA. Österreichischer Frauenthesaurus. Wien: Österr. Staatsdr. Materialien zur Förderung von Frauen in der Wissenschaft. 5.

Koch, Ulrike; Zschokke, Anna 2014: Was bleibt? Grenzen und Möglichkeiten eines queer-feministischen Archivs im Internet. In: Femina politica 23, 2014, H. 2, S. 34-46.

Mayr, Philipp 2006: Thesauri, Klassifikationen \& Co - die Renaissance der kontrollierten Vokabulare? In: Vom Wandel der Wissensorganisation im Informationszeitalter (Festschrift Umstätter). Hauke, Petra (Hrsg.). Humboldt-Universität zu Berlin, Philosophische Fakultät I, Institut für Bibliotheks- und Informationswissenschaft. S. 151-170. http://edoc. hu-berlin.de/miscellanies/festschrift-27533/151/HTML/front.html.

Netzwerk Frauenforschung NRW: Forschungsprojekte. http:// www.netzwerk-fgf.nrw.de/no_cache/genderforschung/forschungsprojekte/forschungsprojektedetailansicht/?tx_p2nwfugf_ pi7[uid] $=247 \& \mathrm{cHash}=1444112452$. 
Projekt META des i.d.a.-Dachverbands. http://www.ida-dachverband.de/ ueber-ida/projekt-meta/.

Schwarzer, Alice; Scheu, Ursula (Hrsg.) 1994. Feministischer Thesaurus. Das Feministische Archiv und Dokumentationszentrum Köln legt den ersten feministischen Thesaurus auf Deutsch vor. Köln: FrauenMediaTurm.

SKOS Simple Knowledge Organization System - Home Page. WC3. http:// www.w3.org/2004/02/skos/.

Women's Information Network of Europe (WINE): https://winenetworkeurope.wordpress.com/.

Zentraleinrichtung zur Förderung von Frauen- und Geschlechterforschung der Freien Universität Berlin: Infrastrukturentwicklung. http://www. zefg.fu-berlin.de/publizieren/Infrastrukturentwicklung/index.html.

Zierold, Marius 2012. XML - aber nicht MARC: VuFind ohne standardisierte Metadatenformate nutzen. Dokumentation der Entwicklung eines zeitgemäßen Bibliothekskataloges für eine wissenschaftliche Spezialbibliothek am Beispiel der Genderbibliothek Berlin. Berlin: HumboldtUniversität zu Berlin, Institut für Bibliotheks- und Informationswissenschaft (unveröffentlichte Magisterarbeit).

1 Vgl. Jank 1991, S. 1418-1421.

2 Jank 1996, S. 1913.

3 Jank 1992, S. 56.

4 Mit dem Begriff Frauen-/Genderinformationseinrichtungen werden im Folgenden die Frauen- und Lesbenarchive bzw. Genderbibliotheken und Dokumentationsstellen vereinfachend zusammengefasst.

5 Mehr Hintergrundinformationen zur Frage „Warum ein feministischer Thesaurus?" bieten die beiden ersten deutschsprachigen Thesauri: a) Schwarzer; Scheu (Hrsg.) 1994, S. 16. b) Klösch-Melliwa; Zach 1996, S. $13 \mathrm{f}$.

6 Aleksander 2014, S. 15 f.

7 Siehe dazu den Beitrag von Margit Hauser und Marius Zierold in diesem Heft.

8 Vgl. Projekt META des i.d.a.-Dachverbands. http://www.ida-dachverband.de/ueber-ida/projekt-meta/.

9 Zentraleinrichtung zur Förderung von Frauen- und Geschlechterforschung der Freien Universität Berlin. Infrastrukturentwicklung. http:// www.zefg.fu-berlin.de/publizieren/Infrastrukturentwicklung/index. html. 
10 Deutschlands Zukunft gestalten. Koalitionsvertrag zwischen CDU, CSU und SPD, 2013, S. 103. https://www.bundesregierung.de/Content/ DE/StatischeSeiten/Breg/koalitionsvertrag-inhaltsverzeichnis.html.

11 Exemplarisch sei an dieser Stelle das EU-Projekt FRAGEN (FRAmes on GENder) erwähnt, in dessen Verlauf eine Datenbank für zentrale Quellentexte der Neuen Frauenbewegung eingerichtet wurde, die mehr als 250 digitalisierte Texte aus mehr als 29 Ländern beinhaltet. Vgl. dazu Jong; Meulmeester; Vriend 2013, S. 80.

12 Women's Information Network of Europe (WINE): https://winenetworkeurope.wordpress.com/.

13 ATGENDER: The European Association for Gender Research, Education and Documentation: http://www.atgender.eu/.

14 EIGE, Resource \& Documentation Centre (RDC): http://eige.europa. $\mathrm{eu} /$ content/rdc.

$15 \mathrm{Vgl}$. EIGE, Agenda des Meetings vom 25.-26. Februar 2015 in Brüssel. http://eige.europa.eu/sites/default/files/Draft-Agenda-EIGERDCmeeting\%20on\%20glossary\%20and\%20thesaurus-16Feb2015.pdf.

$16 \mathrm{Vgl}$. Konferenz der Einrichtungen für Frauen- und Geschlechterstudien im deutschsprachigen Raum (KEG): Protokolle aus den Arbeitsgruppen der KEG 2014: http://www.genderkonferenz.eu/downloads/2014/ AG_Protokolle KEG 2014_30_4_14.pdf.

17 Das Verfahren wird in der bisher unveröffentlichten Magisterarbeit von Marius Zierold beschrieben, die der Verfasserin zur Verfügung gestellt wurde. Vgl. Zierold 2012, S. 30.

$18 \mathrm{Vgl}$. Bertram 2005, S. 228.

19 Mayr 2006, S. 153.

20 Vgl. Klösch-Melliwa; Zach 1996, S. 17.

$21 \mathrm{Vgl}$. Schwarzer; Scheu (Hrsg.) 1994.

$22 \mathrm{Vgl}$. Drenthe; Sommen; Boere 1992.

23 Vgl. Boere; Drenthe 1998.

24 Im verbreiteten Datenbanksystem FAUST können beispielsweise Thesauri komfortabel zentral verwaltet und Vorzugsbenennungen oder Relationen verändert werden, ohne anschließend die Indexierung einzelner Titel korrigieren zu müssen. Ebenso können Schlagworte in Indexlisten leicht angeglichen werden (z.B. Zusammenführung von Singular und Pluralformen).

25 E-Mail vom 22. Februar 2015 in welcher Marianne Boere von Atria (Amsterdam) Fragen zum EWT beantwortet und Empfehlungen für den i.d.a.-Dachverband ausgesprochen hat.

$26 \mathrm{Im}$ Zusammenhang mit dem Wissenschaftsportal vascoda wurden im Projekt KoMoHe zahlreiche kontrollierte Vokabulare über Crosskonkor- 
danzen verbunden. Vgl. Gesis-Forschung: http://www.gesis.org/forschung/drittmittelprojekte/archiv/komohe/.

27 Exemplarisch seien hier zwei Open Source Tools für die Erarbeitung und Visualisierung von mehrsprachigen Thesauri im SKOS-Modell genannt: a) ThManager http://thmanager.sourceforge.net/ und b) VocBench http://www.w3.org/2001/sw/wiki/VocBench.

28 Die Website des TSG ist offline, aber das Projekt wurde im Netzwerk Frauenforschung NRW beschrieben: http://www.netzwerk-fgf.nrw.de/ no_cache/genderforschung/forschungsprojekte/forschungsprojektede tailansicht/?tx_p2nwfugf_pi7[uid]=247\&cHash=1444112452.

29 Vgl. SKOS Simple Knowledge Organization System - Home Page. WC3. http://www.w3.org/2004/02/skos/.

$30 \mathrm{Vgl}$. Koch; Zschokke 2014, S. 24-46. 